\title{
Small-divergence semiconductor lasers by plasmonic collimation
}

\section{Citation}

Yu, Nanfang, Jonathan Fan, Qi Jie Wang, Christian Pflügl, Laurent Diehl, Tadataka Edamura, Masamichi Yamanishi, Hirofumi Kan, and Federico Capasso. 2008. "Small-Divergence Semiconductor Lasers by Plasmonic Collimation." Nature Photonics 2 (9): 564-70. https:// doi.org/10.1038/nphoton.2008.152.

\section{Permanent link}

http://nrs.harvard.edu/urn-3:HUL.InstRepos:41371334

\section{Terms of Use}

This article was downloaded from Harvard University's DASH repository, WARNING: This file should NOT have been available for downloading from Harvard University's DASH repository.

\section{Share Your Story}

The Harvard community has made this article openly available.

Please share how this access benefits you. Submit a story.

Accessibility 


\title{
Small-divergence semiconductor lasers by plasmonic collimation
}

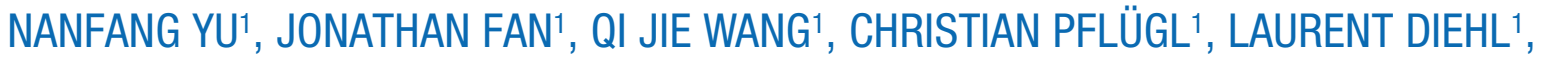 \\ TADATAKA EDAMURA ${ }^{2}$, MASAMICHI YAMANISHI ${ }^{2}$, HIROFUMI KAN² AND FEDERICO CAPASS0 ${ }^{1 *}$
}

${ }^{1}$ School of Engineering and Applied Sciences, Harvard University, Cambridge, Massachusetts 02138, USA

${ }^{2}$ Central Research Laboratory, Hamamatsu Photonics K.K., Hamamatsu 434-8601, Japan

*e-mail: capasso@seas.harvard.edu

Surface plasmons offer the exciting possibility of improving the functionality of optical devices through the subwavelength manipulation of light. We show that surface plasmons can be used to shape the beams of edge-emitting semiconductor lasers and greatly reduce their large intrinsic beam divergence. Using quantum cascade lasers as a model system, we show that by defining a metallic subwavelength slit and a grating on their facet, a small beam divergence in the laser polarization direction can be achieved. Divergence angles as small as $2.4^{\circ}$ are obtained, representing a reduction in beam spread by a factor of 25 compared with the original 9.9- $\mu \mathrm{m}$-wavelength laser used. Despite having a patterned facet, our collimated lasers do not suffer significant reductions in output power ( $\sim 100 \mathrm{~mW}$ at room temperature). Plasmonic collimation provides a means of efficiently coupling the output of a variety of lasers into optical fibres and waveguides, or to collimate them for applications such as free-space communications, ranging and metrology.

\section{INTRODUCTION}

The divergence angle $\theta$ of a beam produced by an edge-emitting semiconductor laser is diffraction-limited to a value $\theta \approx \arcsin (\lambda / T)$ in the plane normal to the waveguide layers, where $\lambda$ is the laser wavelength and $T$ is the thickness of the waveguide core. A similar expression holds for the divergence angle parallel to the layers. In the case of mid-infrared quantum cascade lasers (QCLs), $T$ is limited to a few micrometres and $\theta$ typically ranges from 40 to $80^{\circ}$ (full-width at half-maximum, FWHM) in the polarization direction ( $z$-axis, Fig. 1a), which is normal to the waveguide layers. Conventionally, divergent beams from semiconductor lasers are focused or collimated with lenses or curved mirrors, which usually require meticulous optical alignment. There are a limited number of other methods, including incorporating a micro-machined lens or horn antenna onto the laser facet ${ }^{1,2}$ and using tapered laser waveguides with laterally expanded ends $s^{3,4}$. It is not practical to suppress the vertical divergence by simply growing thick laser active cores; such devices would require unrealistically high voltages for operation and would have heat dissipation problems.

Plasmonic nanostructures known as resonant optical antennas ${ }^{5}$ have been successfully used to modify the near-field of semiconductor lasers by making it possible to generate highintensity nanoscale $(\leq 100 \mathrm{~nm})$ spots $^{6-8}$. Here we show that suitable metallic structures with subwavelength features can be effectively exploited to directly design the far-field of semiconductor lasers and in particular to greatly reduce their beam divergence. Wavefront engineering of laser beams using plasmonic structures or more generally metamaterials can have far-reaching implications for future developments in laser science and technology.

Previously, it has been demonstrated that a plasmonic aperture-groove structure defined in a suspended metal film can collimate incident light ${ }^{9}$. There have been subsequent theoretical and experimental studies of this aperture-groove structure ${ }^{10-14}$, but there have been very few attempts to integrate this structure into an active device ${ }^{15-17}$.

\section{RESULTS}

\section{CONCEPT OF THE SLIT-GRATING PLASMONIC COLLIMATOR}

In our work, a small beam divergence in the vertical direction of QCLs is realized by coupling the outgoing laser radiation through a slit into the surface plasmon (SP) modes of a metallic grating on the laser facet; see Fig. 1a for a schematic of the device. The slit is designed to efficiently couple the laser output into SP waves, and the grating functions as a periodic ensemble of scatterers that coherently radiate the energy of the SP waves into the far-field, leading to increased intensity in a narrow vertical angle. Physically, the resulting collimation can be described as an interference effect, with the metallic structure acting like an antenna array. In summary, the far-field of our QCLs in the vertical direction no longer corresponds to the diffraction from an aperture defined by the waveguide core in the original device, but rather to the interference pattern created by the large number of grooves that span the laser facet. Because mid-infrared SPs are capable of propagating over large distances (at least a few hundred micrometres $)^{18}$, the divergence angle is greatly reduced. Note that the period of the grating $\Lambda$ is designed to match the SP wavelength of the patterned metal surface (second-order grating), so that the beam is normal to the laser facet in the far-field. Also note that the grating is one-dimensional (1D) and hence the laser beam is collimated in one direction ( $z$-axis, Fig. 1a).

In order to yield a small divergence angle, high optical power throughput, and small 'optical background' (defined as the 

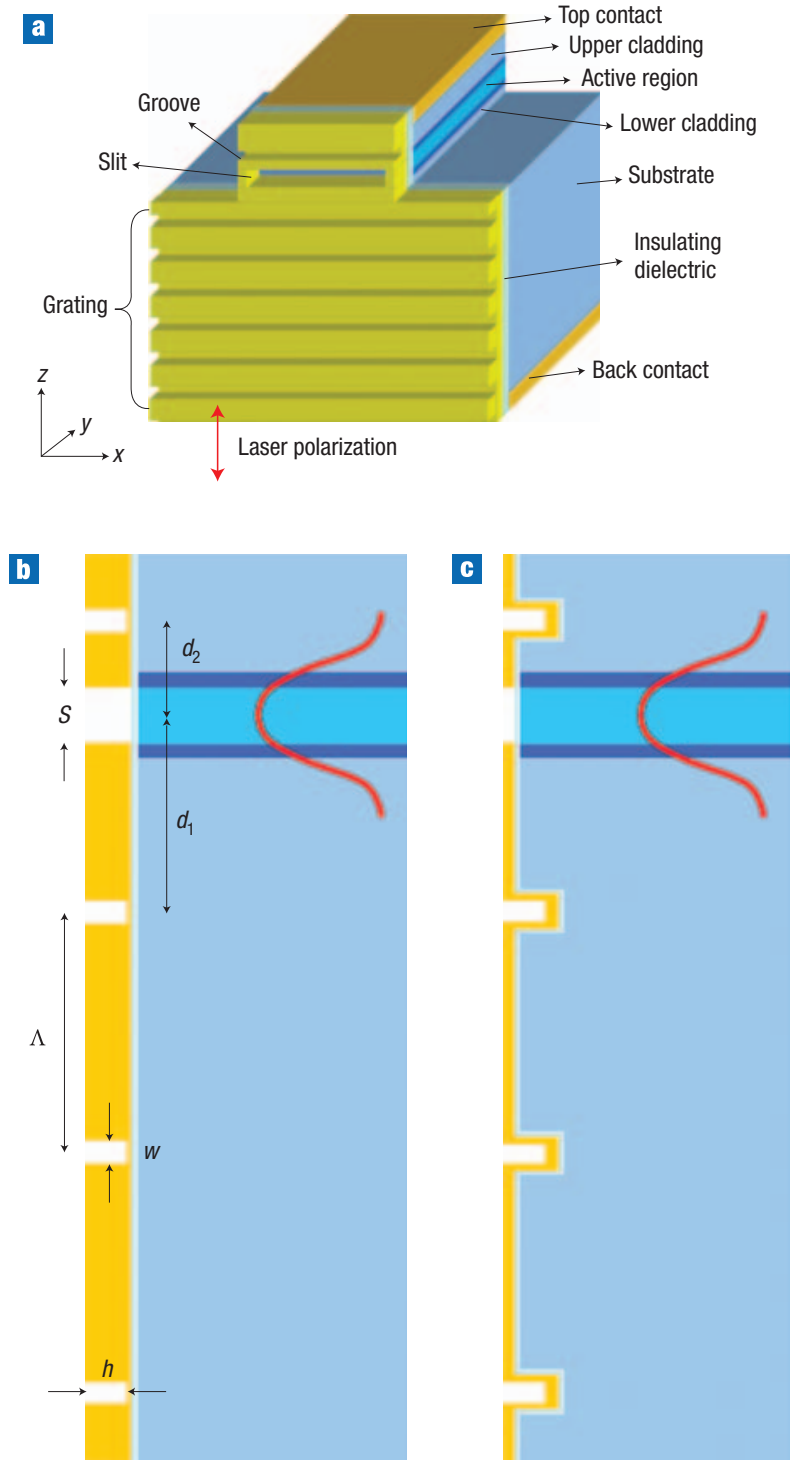

Figure 1 Schematic of the small divergence laser and the two designs. a, Schematic of the small divergence laser, which comprises a QCL and a metallic slit-grating structure defined on its facet. b,c, Cross-sections of the two device designs. In the first design the slit and grooves are defined in a 1.7- $\mu \mathrm{m}$-thick metal film on the laser facet (b). In the second design the grooves are sculpted into the laser facet and a 400-nm-thick layer of metal is added to cover the patterned facet (c). A 200-nm-thick dielectric layer is located between the laser facet and the metal film in both designs for electrical insulation. Only the grooves near the slits are shown in $\mathbf{b}$ and $\mathbf{c}$. Geometric parameters in $\mathbf{b}$ : $s$, width of the slit; $\Lambda$, period of the grooves; $w$ and $h$, width and depth of each groove; $d_{1}$ and $d_{2}$, distances between the slit and the first groove on the substrate side of the laser facet and between the slit and the first groove on the top cladding side of the laser facet, respectively. The optimized values for a laser wavelength of $9.9 \mu \mathrm{m}$ and a grating with 15 grooves are $s=2 \mu \mathrm{m}, \Lambda=8.9 \mu \mathrm{m}, w=0.8 \mu \mathrm{m}, h=1.5 \mu \mathrm{m}$, $d_{1}=7.3 \mu \mathrm{m}$ and $d_{2}=3.5 \mu \mathrm{m}$.

intensity outside the main lobe of the far-field distribution), several geometric parameters of the slit-grating structure need to be optimized, including the width of the slit, the period and number of grooves, the width and depth of each groove, and the distance between the slit and the nearest groove $\left(d_{1}\right.$, Fig. $\left.1 \mathrm{~b}\right)$. The physical considerations used to choose these parameters are discussed in the Methods section.

\section{SIMULATIONS OF THE SLIT-GRATING PLASMONIC COLLIMATOR}

We performed systematic two-dimensional (2D) simulations using COMSOL Multiphysics to help design a slit-grating structure with optimal beam collimation characteristics. The optimized parameters for the $\lambda_{\mathrm{o}}=9.9 \mu \mathrm{m}$ QCLs are given in the caption of Fig. 1. We consider two designs (Fig. 1b,c). In the first design (Fig. 1b), the coating on the laser facet comprises an insulating alumina thin film and a thick gold film. The slit and grooves are defined in the thick gold film. The second design (Fig. 1c) consists of grooves that are sculpted directly into the bare laser facet first, followed by deposition of the insulating and metallic layers and then the opening of the slit. Simulations show that the two designs yield similar performance in beam divergence and power throughput. However, an advantage of the second design is that the gold film thickness is limited only by the skin depth of gold; in our wavelength range, a thickness of $400 \mathrm{~nm}$ is sufficient to prevent SP waves from interacting with the alumina and the semiconductor, thus minimizing optical losses. In addition, grooves defined in the semiconductor show less roughness compared with those sculpted into the metal, which helps reduce the background in the far-field pattern. In consideration of the above, the second design is more suitable for applications in which lasers operate at long wavelengths, such as QCLs. Simulation and experimental results are therefore presented only for the second design. (See Supplementary Information for results for the first design).

Figure 2a shows a simulation of the intensity distribution for an unpatterned $\lambda_{\mathrm{o}}=9.9 \mu \mathrm{m}$ QCL, demonstrating the large divergence of the original device. Figure $2 \mathrm{~b}$ shows a simulation of the intensity distribution for a $\lambda_{\mathrm{o}}=9.9 \mu \mathrm{m}$ QCL patterned with a slit-grating structure of the second design. The grating has 15 grooves, and the simulation shows a central beam and many weaker side beams emerging from the laser facet. Figure $2 \mathrm{c}$ is the calculated far-field intensity distribution for the patterned device obtained using a near-field to far-field transformation algorithm. The central beam has a vertical divergence angle slightly smaller than $4^{\circ}$, and the optical background is relatively uniform as a function of angle and has an average intensity that is $<10 \%$ of the central lobe peak intensity. Figure $2 \mathrm{~d}$ shows the electric field magnitude distribution around the slit and the first few grooves of the grating for the patterned device. The surface waves are strongly localized to the patterned facet of the device, indicating a strong coupling between the two due to Bragg diffraction; this is significantly different from the weak coupling of mid-infrared surface waves to a flat 'planar' metallic surface. The latter case is demonstrated in Fig. 2e, which shows that surface waves are loosely attached to the metal-air interface for a device with just the slit aperture and without the grating.

\section{FAR-FIELD MEASUREMENTS}

We fabricated the two devices depicted in Fig. 1b,c; details of the fabrication procedure are provided in the Methods section. Figure 3a,d shows scanning electron microscope (SEM) images of two $\lambda_{\mathrm{o}}=9.9 \mu \mathrm{m}$ QCLs before and after patterning the slitgrating structure of the second design. The $2 \mathrm{D}$ far-field intensity distributions measured before and after patterning of the plasmonic slit-grating structure are presented in Fig. 3b,c,e,f, demonstrating a strong reduction of the beam divergence in the vertical direction. The slight curvature of the far-field patterns in Fig. 3e,f is an edge effect; the finite size of the slit aperture in the lateral direction introduces lateral components in the SP wavevector $\left(k_{\mathrm{sp}}\right)$, reducing the vertical 

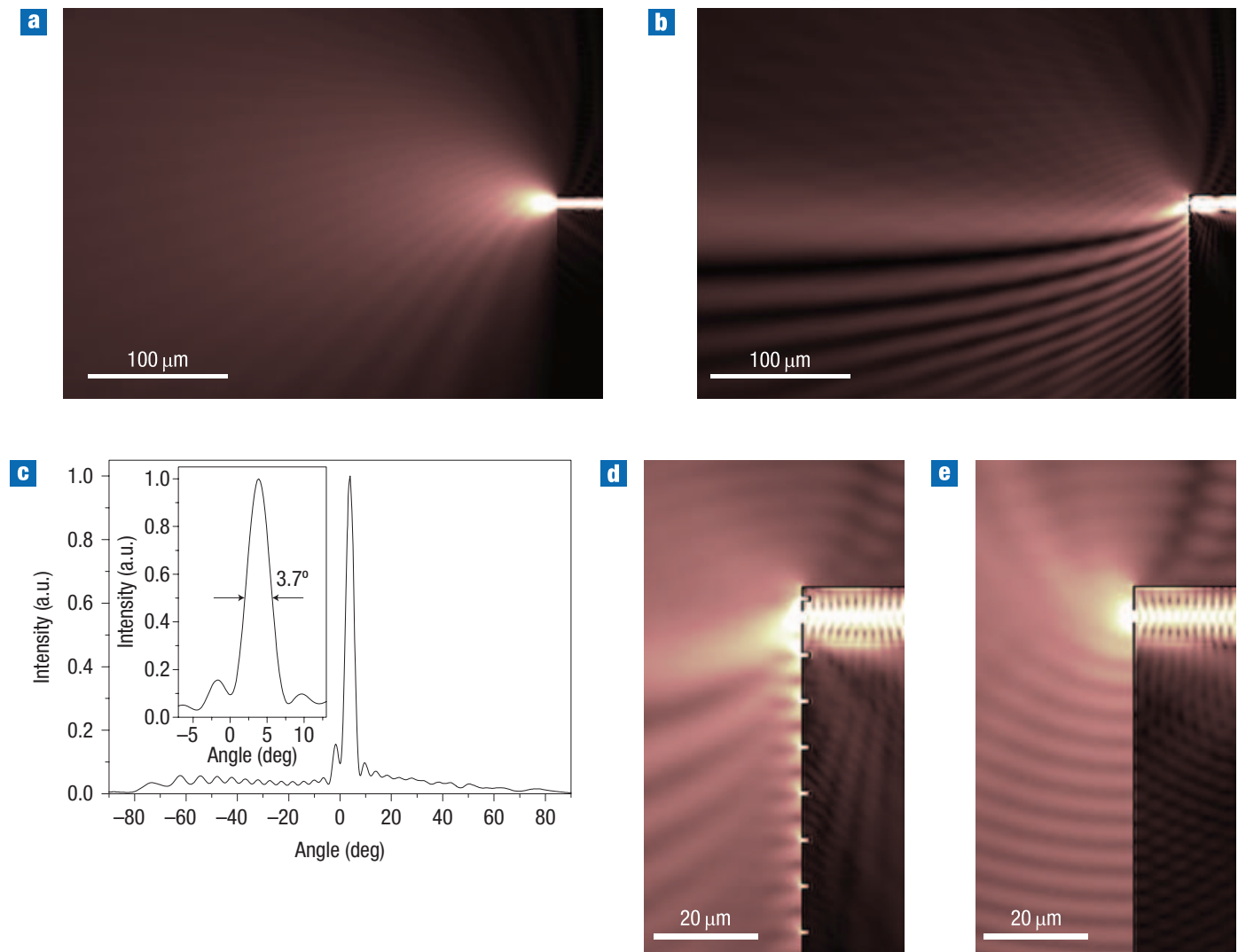

Figure 2 Simulations. a, 2D simulation of the intensity distribution of an original unpatterned QCL lasing at $\lambda_{0}=9.9 \mu \mathrm{m}$. The simulation plane is perpendicular to the laser waveguide layers and along the middle of the waveguide. $\mathbf{b}, 2 \mathrm{D}$ simulation of the intensity distribution of a small-divergence QCL of the second design lasing at $\lambda_{0}=9.9 \mu \mathrm{m}$. In this simulation, there are 15 grooves between the slit and the bottom electrical contact. The thickness of the gold film is $400 \mathrm{~nm}$; other geometric parameters are the optimized values presented in the caption of Fig. 1. c, Calculated far-field intensity distribution of the device shown in b; inset: enlarged view of the central lobe. d, Simulation of the magnitude of the electric field around the slit and the first seven grating grooves. e, Simulation of the electric field magnitude distribution for a $\lambda_{0}=9.9 \mu \mathrm{m}$ QCL with the slit aperture and without the grating.

components $k_{\mathrm{sp} \perp}$ compared with the case of an infinitely wide slit, where $k_{\mathrm{sp} \perp}=k_{\mathrm{sp}}$. This produces a wavevector mismatch $\left(k_{\mathrm{sp} \perp}<2 \pi / \Lambda\right)$, leading to a small beam deflection towards the top of the device. The line scans of the $2 \mathrm{D}$ far-field emission patterns in the laser polarization direction are provided in Fig. $4 \mathrm{a}-\mathrm{c}$, which show that the divergence angle is reduced from $\sim 62$ to $2.9^{\circ}$ for the device with 20 grating grooves, and from $\sim 63$ to $2.4^{\circ}$ for the device with 24 grating grooves. For the device with 24 grating grooves, the grooves cover the entire laser facet from the slit to the bottom electrical contact. The line scans also show that the average intensity of the background of the two devices is less than $10 \%$ of the peak value of the central lobe, which is an improvement compared with the devices of the first design (see Supplementary Information). This is due to the improved quality of the grating structure when it is sculpted directly into the semiconductor. Note that the measured far-field intensity profile is reproducible in multiple devices, and that the metallic collimator is not damaged after high peak power operation. The beam quality factor $\left(\mathrm{M}^{2}\right.$ factor $)$ of the device with 24 grating grooves is determined to be $\sim 2.5$ based on measurements of the variation of the vertical beam waist along the propagation direction. As the reduced beam divergence results from an antenna effect, as previously discussed, we can use as a figure of merit the concept of antenna directivity for the performance of the plasmonic structure ${ }^{19}$. The directivity is used here to characterize collimation in the vertical direction, and is defined as $D=10 \log _{10}\left(2 \pi I_{\text {peak }} / I_{\text {total }}\right)$, where $I_{\text {peak }}$ is the far-field peak intensity and $I_{\text {total }}$ is the total intensity under the vertical beam profile. We find that the directivity $D$ is $\sim 17.7$ and $\sim 18.3 \mathrm{~dB}$ for the two devices with 20 and 24 grooves, respectively, whereas it is only $\sim 7.2 \mathrm{~dB}$ for the original unpatterned lasers.

The QCLs used in our experiment have only one lobe in the lateral direction ( $x$-axis, Fig. 1a) corresponding to the $\mathrm{TM}_{00}$ lateral mode. The lateral beam divergence after definition of the slit-grating structures is nearly equal to that of the original lasers (Fig. 4d); this is because the grating is a $1 \mathrm{D}$ structure, so beam divergence is greatly reduced only in the direction perpendicular to the grooves.

\section{LIMITS ON BEAM DIVERGENCE}

One of the limitations on the narrowest vertical divergence angle $\left(\theta_{\min }\right)$ that can be achieved is the size of the laser facet patterned with grooves. Calculations show that $\theta_{\min }$ scales in inverse proportion to the number of grooves, as expected from an interference effect. For a laser wavelength of $9.9 \mu \mathrm{m}$, simulations indicate that for 15 grooves spanning $\sim 130 \mu \mathrm{m}$ on the laser facet in the vertical direction, $\theta_{\text {min }}$ is $\sim 3.7^{\circ}$, but this drops to $\sim 1.0^{\circ}$ for 70 grooves spanning $\sim 630 \mu \mathrm{m}$. In the latter case, the width of the grooves $w$ needs to be slightly smaller than in the former, to allow SPs to propagate across all the grooves. Another fundamental 


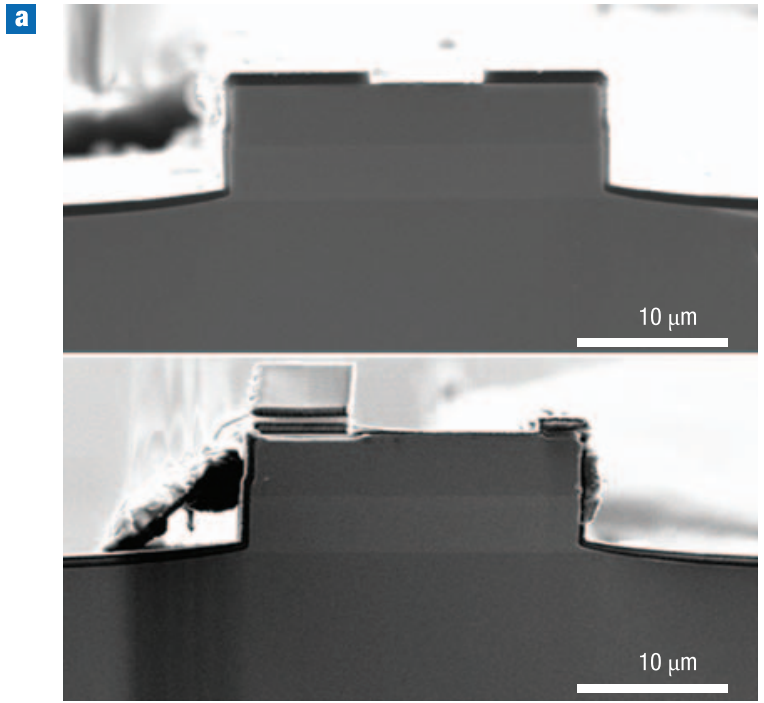

d

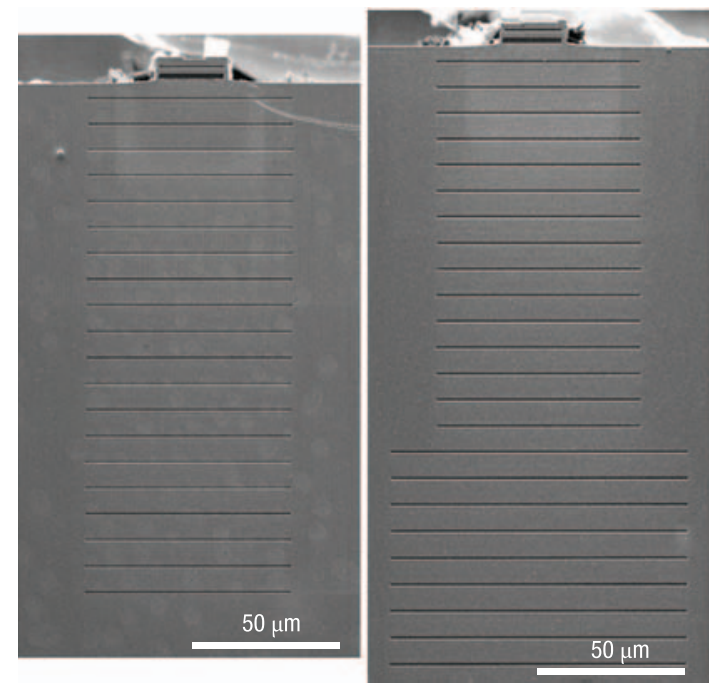

b
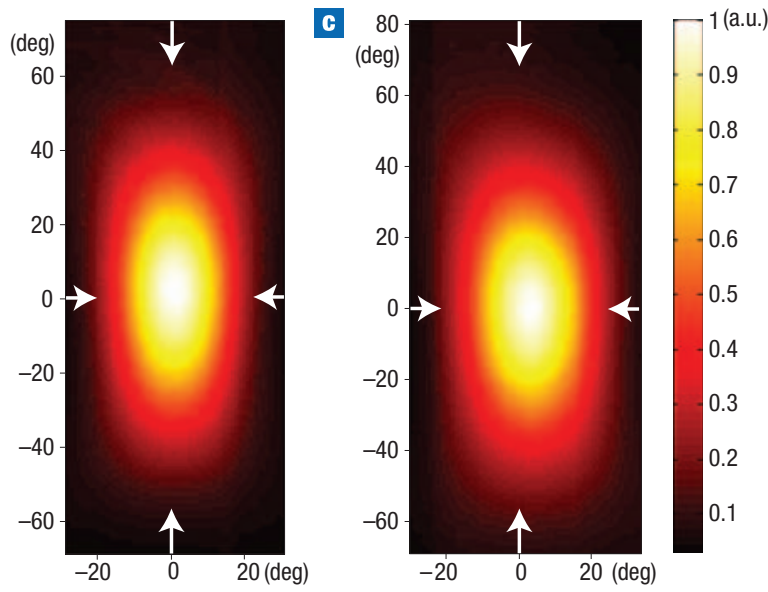

$\theta$

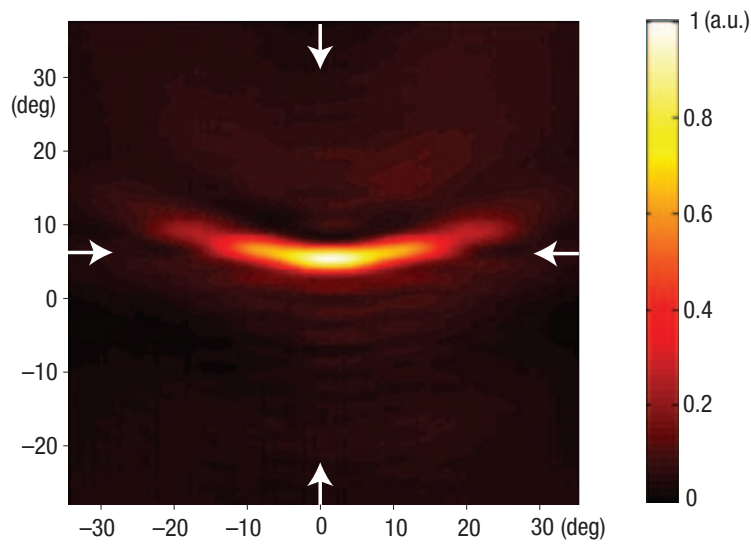

f

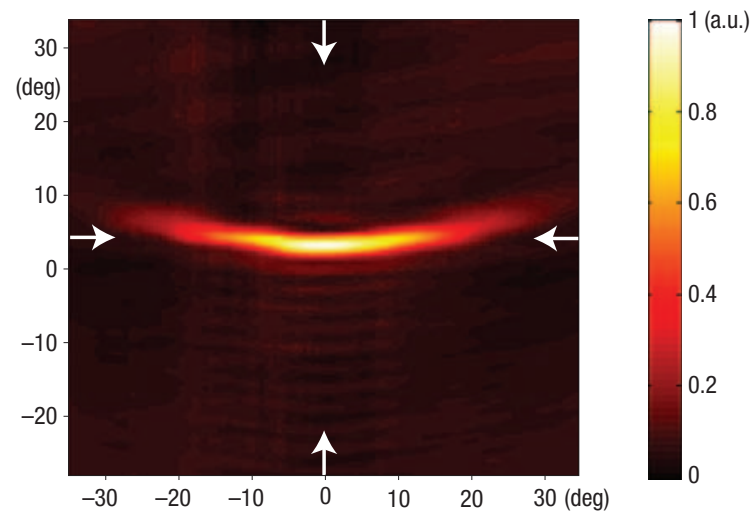

Figure 3 Experimental results of two devices of the second design. a, SEM images of the facets of two original unpatterned QCLs lasing at $\lambda_{0}=9.9 \mu \mathrm{m}$. Device A (upper panel) has a ridge that is $25 \mu \mathrm{m}$ wide and $2 \mathrm{~mm}$ long; device B (lower panel) has a ridge that is $22 \mu \mathrm{m}$ wide and $2 \mathrm{~mm}$ long. b,c, Measured $2 \mathrm{D}$ far-field intensity distributions of the original unpatterned devices $A$ and $B$, respectively. $\mathbf{d}$, SEM images of the facets of devices $A$ and $B$ patterned with the slit-grating structure of the second design. Device A (left panel) has 20 grating grooves. Device B (right panel) has 24 grating grooves. e,f, Measured 2D far-field intensity distributions of devices $A$ and $B$ patterned with the slit-grating structure, respectively.

limitation on $\theta_{\min }$ is the width of the spectrum of the laser in use. According to simulations, a change of the laser wavelength by $0.1 \mu \mathrm{m}$ in the mid-infrared region leads to a shift in the position of the central lobe by $\sim 1^{\circ}$. This is believed to be the main reason why the measured divergence angles of all devices with the slitgrating structure are $10-20 \%$ larger than those in the simulations, as the lasers used are multimode and have a bandwidth of $\sim 0.05-0.1 \mu \mathrm{m}$. A single-mode laser with a large facet area patterned with the slit-grating structure is capable of vertical collimation with a divergence angle smaller than $1^{\circ}$.

\section{LIGHT OUTPUT VERSUS CURRENT CHARACTERISTIC}

Figure 5 shows the light output versus current characteristics before and after defining the slit-grating structure, demonstrating a maximum output power of $\sim 100 \mathrm{~mW}$. The slope efficiency (SE) of the light/current characteristics of the patterned devices is 

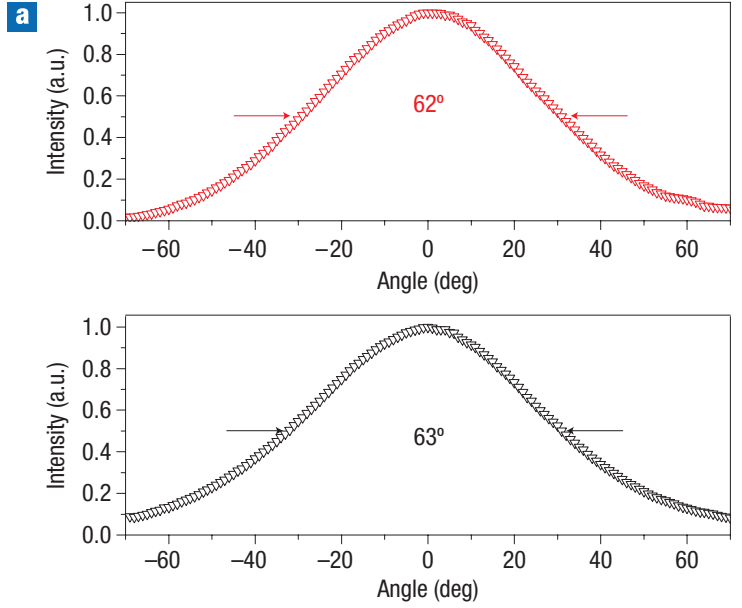

$\mathbf{G}$

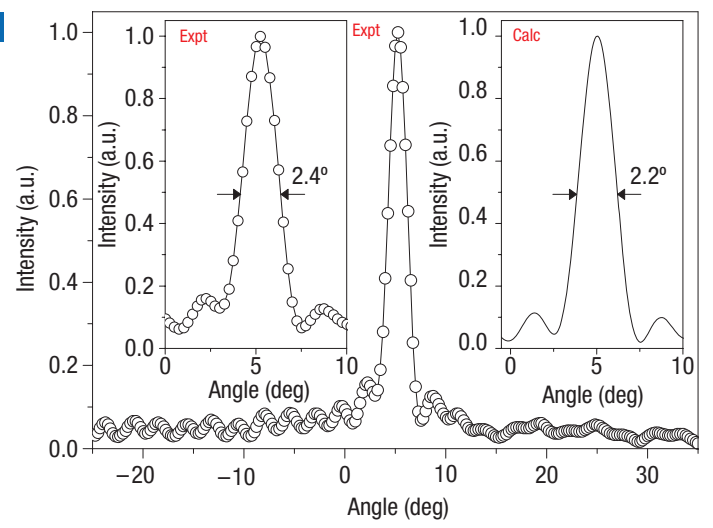

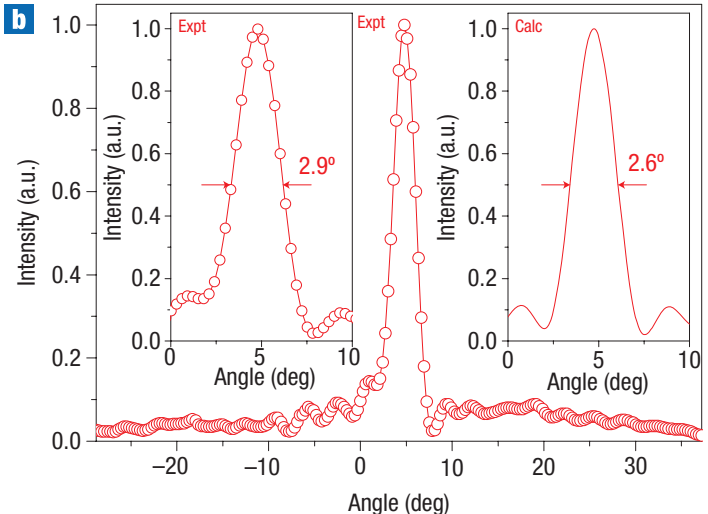
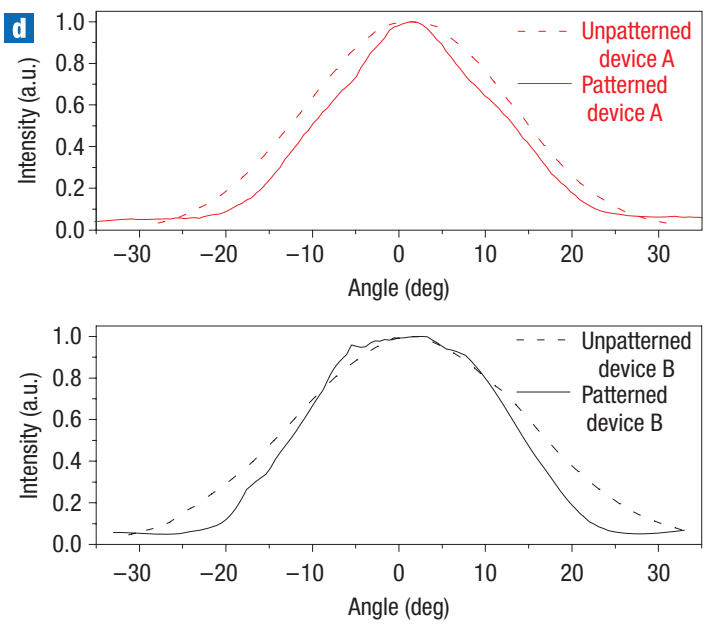

Figure 4 Line scans of the 2D far-field intensity distributions. a, Upper and lower panels are vertical line scans of Fig. 3b,c along the arrows, respectively. b, Vertical line scan of Fig. 3e along the arrows. Left and right insets are the enlarged views of the experimental and calculated central lobe of the distribution, respectively. c, Vertical line scan of Fig. $3 f$ along the arrows. Left and right insets are the enlarged views of the experimental and calculated central lobe of the distribution, respectively. d, Upper panel: solid and dashed curves are horizontal line scans of Fig. 3b,e along the arrows, respectively. Lower panel: solid and dashed curves are horizontal line scans of Fig. 3c,f along the arrows, respectively.

determined primarily by the efficiency of the plasmonic grating to couple SP waves into propagating waves. We found that larger SEs correlate with gratings with a larger number of grooves; devices with 24 grooves had an SE of $\sim 180 \mathrm{mWA}^{-1}$, which is $\sim 90 \%$ of that of the original laser without a collimator. Devices processed the same way but with only 15 grooves had an SE of only $\sim 120 \mathrm{~mW} \mathrm{~A}^{-1}$, indicating incomplete coupling of surface waves into the far-field. In an optimized design one expects only a small reduction of the SE due to the low SP losses in the midinfrared, implying a small change in the effective reflectivity of the facet. The increase of the threshold current for devices of the second design (Fig. 5) is likely due to a leakage current path along the alumina layer coated on the laser. An improved fabrication process using a thicker alumina insulating layer would likely greatly reduce this problem.

\section{DISCUSSION}

In summary, we have demonstrated, by a combination of experiments and modelling, that the integration of a suitably designed slit-grating structure on the facet of QCLs reduces the vertical beam divergence angle by a factor of $\sim 25$ down to $2.4^{\circ}$, while preserving a high output power comparable to that of the unpatterned lasers. The plasmonic collimation concept can be extended to edge-emitting and surface-emitting semiconductor lasers from the visible to the near-infrared. For the specific case of TE-polarized sources, like most diode lasers, slit and grating structures perpendicular to the polarization direction may be implemented.

It is interesting to note the differences between our laser with the plasmonic collimator and a conventional second-order grating surface-emitting laser. Although the latter also achieves small beam divergence in one direction and high output power ${ }^{20}$, many applications require an edge- rather than surface-emitting geometry. The advantage of the plasmonic collimation scheme lies in its flexibility; it can be upgraded to a $2 \mathrm{D}$ periodic structure that can collimate light in both the vertical and lateral directions, thus achieving complete beam collimation, and can be modified to control the polarization of the laser. We recently designed a mid-infrared wavelength collimator consisting of an aperture centred on the QCL active region and a metallic grating 


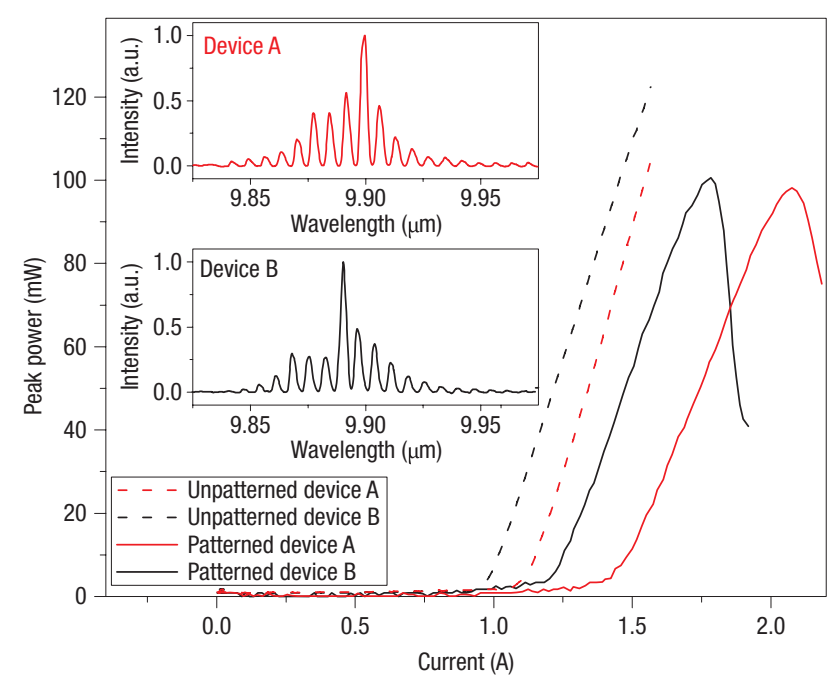

Figure 5 Light output versus current characteristic taken before and after defining the slit-grating structure. The dashed and solid curves are for the original unpatterned devices and those with the plasmonic collimator, respectively. The red and black curves correspond to devices $A$ and $B$, respectively. Inset: laser spectra of devices $A$ (upper panel, $I=1.8 \mathrm{~A}$ ) and $\mathrm{B}$ (lower panel, $I=1.5 \mathrm{~A}$ ). In all the measurements, the lasers were operated at room temperature in pulsed mode with an $80-\mathrm{kHz}$ repetition rate and $125-\mathrm{ns}$ pulse duration.

of concentric half-rings. Simulations show that this structure is able to radially generate SPs on the laser facet and that a suitable grating consisting of 20 equally spaced gold half-rings can produce a beam with divergence angles (FWHM) of 2.7 and $3.2^{\circ}$ in the vertical and lateral directions, respectively, at $\lambda=8.06 \mu \mathrm{m}$. Preliminary experiments on this structure confirm collimation in both directions with divergence angles comparable to the calculated ones.

Future plans also include further reducing the vertical beam divergence by using single-mode QCLs with a larger patterned area. We also plan to perform detailed near-field imaging studies and $3 \mathrm{D}$ simulations of the SP waves on the patterned laser facets, which will help us further elucidate the interaction between the SP waves and the slit-grating structure.

Finally we wish to point out that plasmonic nanostructures (and more generally metamaterials) can be built on the facet of any type of semiconductor laser and also on fibre lasers to achieve complex wavefront engineering. Specific beam shaping and polarization control for a variety of applications can be envisioned.

\section{METHODS}

\section{DESIGN CONSIDERATIONS FOR THE SLIT-GRATING PLASMONIC COLLIMATOR}

The vertical width of the slit ( $s$, Fig. 1b) needs to be subwavelength with respect to the free-space wavelength $\lambda_{\mathrm{o}}$ to efficiently couple incoming radiation from the laser into the SP modes of the grating structure. The optimized value of $s$ is found to be close to the active core thickness ( $\sim 2 \mu \mathrm{m}$ for $\lambda_{\mathrm{o}}=9.9 \mu \mathrm{m}$ QCLs). A narrower slit strongly backscatters the incoming light and reduces power throughput, but a wider slit does not efficiently couple incoming light into the SP modes and yields a larger background.

It is preferable for the number of grooves $N$ to be as large as possible. Simulations show that the peak intensity in the far-field scales approximately with the square of $N$ up to $N \approx 30$, as expected from an interference effect; the divergence angle scales inversely proportional to $N$ at least up to $N \approx 70$. For $N>30$, the effect of loss gives rise to a weaker variation of the peak intensity with $N$.
Wavevector conservation in the plane of the laser facet requires $k_{\text {sp-grating }}-2 \pi / \Lambda=k_{\mathrm{o}} \sin \theta$, where $k_{\mathrm{sp}-\text { grating }}$ is the wavevector of the SPs on the patterned metal surface, $\Lambda$ is the grating period, $k_{\mathrm{o}}$ is the free-space wavevector, and $\theta$ is the angle between the latter and the normal to the facet. $\Lambda$ is designed to satisfy the condition for second-order gratings for the SPs: $\Lambda=2 \pi / k_{\text {sp-grating. }}$. Thus the re-emitted light has a wavevector normal to the laser facet $(\theta=0)$. At the same frequency, the wavevector of the SPs on a metal surface patterned with a grating $\left(k_{\mathrm{sp} \text {-grating }}\right)$ is larger than the wavevector of the SPs on a planar metal surface $\left(k_{\mathrm{sp}-\text { planar }}\right)^{13}$, which is almost equal to the free-space wavevector $k_{\mathrm{o}}$ in the mid-infrared region of the spectrum. As a result, the grating period $\Lambda(=8.9 \mu \mathrm{m})$ should be smaller than the free-space wavelength $\left(\Lambda=2 \pi / k_{\text {sp-grating }}<2 \pi / k_{\text {sp-planar }} \approx 2 \pi / k_{\mathrm{o}}=\lambda_{\mathrm{o}}=9.9 \mu \mathrm{m}\right)$ in order to generate a collimated beam normal to the laser facet. The difference between $\Lambda$ and $\lambda_{\mathrm{o}}$ is a measure of the modification of the SP dispersion curve caused by the grating and the coupling strength between surface waves and the grating.

The width $w$ and depth $h$ of the grooves are two additional important factors to optimize. We find that deep and thin grooves are the most suitable for the purpose of beam collimation. Simulations with thick grooves show that they cannot simultaneously yield a small divergence angle and a small background. Grooves that are thick and deep interact with SPs too strongly, which limits surface wave propagation along the grating to only a few grooves and results in limited beam collimation. On the other hand, thick and shallow grooves yield a large background, meaning that the coupling to SPs is too weak. Note that the width of the grooves should decrease with the increase in the number of grooves to optimize interaction between the SPs and the entire length of the grating.

Coupling of laser radiation to the grating must be optimized in order to maximize the collimated optical power. To ensure this, the distance $d_{1}$ between the slit and the closest groove on the substrate side of the facet must be adjusted to maximize the destructive interference between the reflected SPs from the grating and the SPs that are emerging from the slit and propagating towards the top electrical contact ${ }^{21}$. Our simulation gives $d_{1}=7.3 \mu \mathrm{m}$.

Owing to the limited area of the laser facet, the majority of grooves can only be defined on one side of the slit, between the slit and the bottom electrical contact. However, one or more additional grooves can still be included on the other side of the slit, between the slit and the top electrical contact. These additional grooves are crucial in increasing the intensity of the central lobe and substantially reducing the background in the far-field distribution. Physically, they function as a reflector for the residual surface waves propagating towards the laser top contact, thereby reducing scattering from the latter. In summary, these reflector grooves, combined with the destructive interference condition previously mentioned, allow for our 'asymmetric' design to collimate laser light with similar divergence angle and central lobe intensity, as compared to a 'symmetric' design consisting of an equal number of grooves equally distributed on both sides of the slit.

\section{FABRICATION}

In the first design (Fig. 1b), the device facet was first coated with a layer of electrically insulating alumina, followed by a layer of gold using electron-beam evaporation. Focused ion beam (FIB) milling was then used to define the slitgrating structure in the gold layer. In the second approach (Fig. 1c), the grooves were first defined on the bare device facet using FIB, the insulating alumina and gold films were then deposited, and finally the slit was opened with FIB. A twoangle deposition procedure was used to coat both walls of the grooves. The thicknesses of the alumina and gold layers on the laser facet were $\sim 200 \mathrm{~nm}$ and $\sim 400 \mathrm{~nm}$, respectively, and their thicknesses on the walls of the grooves were $\sim 100 \mathrm{~nm}$ and $\sim 200 \mathrm{~nm}$, respectively.

\section{DEVICES}

The QCLs used in our experiments were operated in pulsed mode at room temperature and lased at $\lambda_{\mathrm{o}} \approx 9.9 \mu \mathrm{m}$. The QCL material was grown by molecular beam epitaxy (MBE) on an InP substrate (n-doped with silicon, $3 \times 10^{18} \mathrm{~cm}^{-3}$ ). The growth process started with a lower InP cladding layer (n-doped with silicon, $1 \times 10^{17} \mathrm{~cm}^{-3}$, thickness $3.5 \mu \mathrm{m}$ ), followed by a waveguide core (thickness $3.16 \mu \mathrm{m}$ ), and ended with an upper InP cladding layer (n-doped with silicon, $1 \times 10^{17} \mathrm{~cm}^{-3}$, thickness $3.5 \mu \mathrm{m}$ ) and contact layers. The waveguide core consisted of an active core (thickness $2.12 \mu \mathrm{m}$ ) sandwiched between two InGaAs cladding layers (n-doped with silicon, $3 \times 10^{16} \mathrm{~cm}^{-3}$, thickness of each layer $0.52 \mu \mathrm{m}$ ). The active core consisted of 35 periods, which were composed of alternating injector regions and four-quantum-well active regions based on a double phonon resonance design ${ }^{22}$. The barrier/quantum well composition was InAlAs/InGaAs lattice-matched to InP. 
The material was processed into ridge waveguides $2-3 \mathrm{~mm}$ long and $10-25 \mu \mathrm{m}$ wide, with a 300 -nm-thick $\mathrm{Si}_{3} \mathrm{~N}_{4}$ insulating layer on the lateral walls of the ridge and a 300-nm-thick gold top contact. The samples were indium-soldered on nickel-/gold-plated copper holders.

\section{MEASUREMENTS}

For the measurement of the QCL far-field, we used a set-up in which the tested device was mounted at the centre of a motorized rotation stage, and a midinfrared mercury-cadmium-telluride detector $10-20 \mathrm{~cm}$ away was scanned across a certain angle to measure the output of the laser. Our measurements were performed with a resolution of $0.25^{\circ}$. Power measurements were carried out with a calibrated power meter. The power meter, which has a metallic collection tube with a diameter of $6.5 \mathrm{~mm}$, was placed within $2 \mathrm{~mm}$ of the lasers, thus collecting power in an angular range of approximately $\pm 60^{\circ}$ with respect to the normal of the laser facet. A Fourier-transform infrared spectrometer was used for spectral measurements.

Received 21 April 2008; accepted 20 June 2008; published 27 July 2008.

\section{References}

1. Lee, A. W. M. et al. High-power and high-temperature THz quantum-cascade lasers based on lenscoupled metal-metal waveguides. Opt. Lett. 32, 2840-2842 (2007).

2. Amanti, M. I., Fischer, M., Walther, C., Scalari, G. \& Faist, J. Horn antennas for terahertz quantum cascade lasers. Electron. Lett. 43, 573-574 (2007).

3. Troccoli, M., Gmachl, C., Capasso, F., Sivco, D. L. \& Cho, A. Y. Mid-infrared ( $\lambda \approx 7.4 \mu \mathrm{m})$ quantum cascade laser amplifier for high-power single-mode emission and improved beam quality. Appl. Phys. Lett. 80, 4103-4105 (2002).

4. Nähle, L., Semmel, J., Kaiser, W., Höfling, S. \& Forchel, A. Tapered quantum cascade lasers. Appl. Phys. Lett. 91, 181122 (2007).

5. Mühlschlegel, P., Eisler, H. -J., Martin, O. J. F., Hecht, B. \& Pohl, D. W. Resonant optical antennas. Science 308, 1607-1609 (2005).

6. Cubukcu, E., Kort, E. A., Crozier, K. B. \& Capasso, F. Plasmonic laser antenna. Appl. Phys. Lett. 89, 093120 (2006)

7. Yu, N. et al. Plasmonic quantum cascade laser antenna. Appl. Phys. Lett. 91 , $173113(2007)$.

8. Yu, N. et al. Bowtie plasmonic quantum cascade laser antenna. Opt. Express 15, $13272-13281$ (2007).

9. Lezec, H. J. et al. Beaming light from a subwavelength aperture. Science 297, 820-822 (2002).
10. Martín-Moreno, L., García-Vidal, F. J., Lezec, H. J., Degiron, A. \& Ebbesen, T. W. Theory of highly directional emission from a single subwavelength aperture surrounded by surface corrugations. Phys. Rev. Lett. 90, 167401 (2003).

11. Baida, F. I., Labeke, D. V. \& Guizal, B. Enhanced confined light transmission by single subwavelength apertures in metallic films. Appl. Opt. 42, 6811-6815 (2003).

12. Akarca-Biyikli, S. S., Bulu, I. \& Ozbay, E. Enhanced transmission of microwave radiation in one-dimensional metallic gratings with subwavelength aperture. Appl. Phys. Lett. 85, $1098-1100$ (2004).

13. Yu, L.-B. et al. Physical origin of directional beaming emitted from a subwavelength slit. Phys. Rev. B 71, 041405(R) (2005).

14. Kim, S., Kim, H., Lim, Y. \& Lee, B. Off-axis directional beaming of optical field diffracted by a single subwavelength metal slit with asymmetric dielectric surface gratings. Appl. Phys. Lett. 90, 051113 (2007).

15. Guo, B., Song, G. \& Chen, L. Plasmonic very-small-aperture lasers. Appl. Phys. Lett. 91, 021103 (2007).

16. Gao, J., Song, G., Gan, Q., Guo, B. \& Chen, L. Surface plasmon modulated nano-aperture verticalcavity surface-emitting laser. Laser Phys. Lett. 4, 234-237 (2007).

17. Hirohata, T., Niigaki, M., Mochizuki, T., Fujiwara, H. \& Kan, H. Near-infrared photocathode using surface plasmon resonance. Jpn J. Appl. Phys. 46, 630-632 (2007).

18. Novotny, L. \& Hecht, B. Principles of Nano-Optics (Cambridge Univ. Press, Cambridge, 2006).

19. Stutzman, W. L. \& Thiele, G. A. Antenna Theory and Design (John Wiley \& Sons, New York, 1981)

20. Pflügl, C. et al. Single-mode surface-emitting quantum-cascade lasers. Appl. Phys. Lett. 86, 211102 (2005).

21. López-Tejeira, F. et al. Efficient unidirectional nanoslit couplers for surface plasmons. Nature Phys. 3 $324-328(2007)$

22. Hofstetter, D. et al. Continuous wave operation of a $9.3 \mu \mathrm{m}$ quantum cascade laser on a Peltier cooler. Appl. Phys. Lett. 78, 1964-1966 (2001).

Supplementary Information accompanies this paper at www.nature.com/naturephotonics.

\section{Acknowledgements}

We acknowledge support from the Air Force Office of Scientific Research (AFOSR MURI on Plasmonics) and the Harvard Nanoscale Science and Engineering Centre (NSEC). This work was performed in part a the Centre for Nanoscale Systems (CNS) at Harvard University, a member of the National

Nanotechnology Infrastructure Network (NNIN), which is supported by the National Science

Foundation under NSF award no. ECS-0335765. CNS is part of the Faculty of Arts and Sciences at

Harvard University. We would like to thank H. Mosallaei for helpful discussions and suggestions. We

acknowledge the contributions of R. Blanchard in the preliminary simulations of the ringshaped collimators.

Author information

Reprints and permission information is available online at http://npg.nature.com/reprintsandpermissions/. Correspondence and requests for materials should be addressed to F.C 